\title{
Ideology of Image Segmentation and Methods Thereof
}

\author{
Varunika Arya ${ }^{1}$, Ranjit Kaur $^{2}$, Amit Goyal $^{3}$ \\ ${ }^{1,3}$ (Department of Electronics and Communication Engineering, MM University-Sadopur, India) \\ ${ }^{2}$ (Department of Electronics and Communication Engineering, Punjabi University-Patiala, India)
}

\begin{abstract}
Image processing has emerged to be the only stand-alone non-human technique for image comprehension. The avant-garde image processing techniques finds their vast relevance in feature extraction, face detection, finger-print detection, microscope imaging, medical imaging, computer vision, remote sensing, etc. Image processing constitutes a systematic procedure of signal processing, computer vision and artificial intelligence leading to application dependent sorting of unique set of image features like intensity, color, texture, etc. Eventually, segmentation of image has surfaced as a crucial milestone in image processing and its success counts in better results of the trailing steps: be it boundary based (edge detection, watershed, etc.), threshold based (histogram, local, global, etc.) or region based segmentation (region growing, merging, splitting, etc.. This paper intends to explicate a methodical study of a range of image segmentation techniques along with various methods to optimize the results of segmentation. In addition to subjective evaluation, a quantitative survey for the evaluation of the segmented results is also extended.
\end{abstract}

Keywords: edge detection, optimization, region growing, segmentation, threshold.

\section{INTRODUCTION}

The perception of a physical phenomenon by a sensing device, often termed as convention image acquisition, is the formative origin of processing it with a digital computer [5] and thus results in digital image processing. Digital image is formed of pixels, each having a particular position and amplitude. Images may be further categorized as binary (with only two levels of intensities either black or white), gray scale (with 256 different levels of intensities varying from black to white) or colored image (each pixel consisting of combination of red, green and blue intensity levels) [13]. Fig.1.shows a binary image and the corresponding pixel detail (Courtesy: MATLAB toolbox). Similar duo for gray scale and colored images are shown in fig.2 and fig.3, respectively.
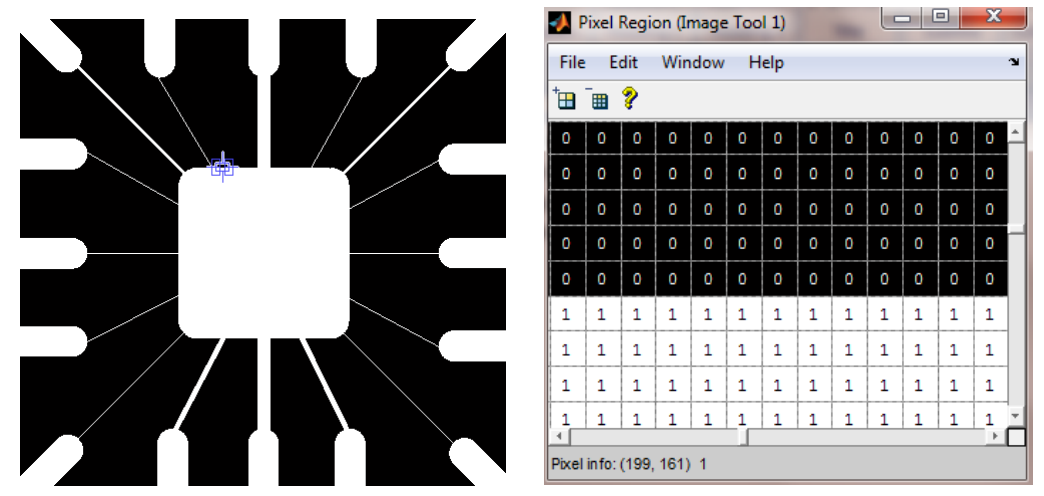

Fig.1: Binary image and the corresponding pixel detail (Courtesy: MATLAB toolbox)

From low-level processes like noise reduction, contrast enhancement and image sharpening to mid-level processing such as segmentation of image, description \& recognition of objects in an image, and finally performing the cognitive higher level functions associated with vision, the growth of image processing as a technical discipline has led to several futuristic applications in medical imagery [7, 15], remote sensing, agricultural applications etc. [11]. Image segmentation forms the heart of image processing and hence its accuracy influences the later stages of processing the image $[3,24]$. The main approach of image segmentation is to partition an image into its constituent 
object to make it easier to analyze. It basically separates the mutually homogeneous regions of interests i.e., assign pixels to a region having common attributes like intensity, color, texture etc. [9].
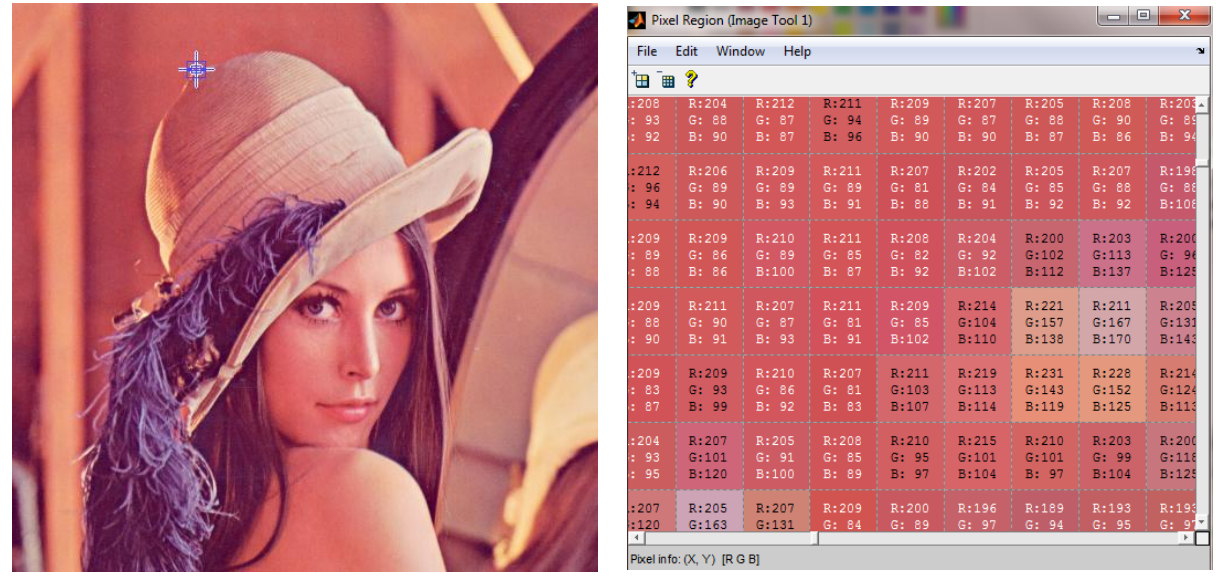

Fig.2. Colored image and the corresponding pixel detail (Courtesy: MATLAB toolbox)
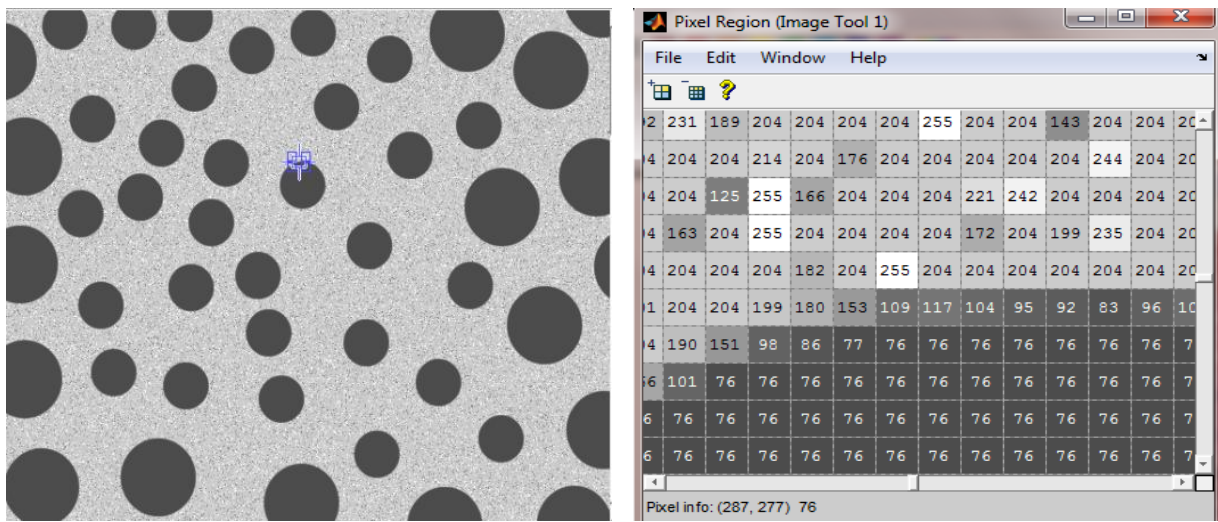

Fig.2: Colored image and the corresponding pixel detail (Courtesy: MATLAB toolbox)

Segmentation plays a fundamental role in the field of image processing, image analysis, and coding with a wide range of applications as object matching, object recognition or object detection. It is also widely used in medical imaging such as MRI, CT scan, etc. [10]. Various sorts of segmentation techniques have been evolved in the past which are categorized on the basis of two traits of the image namely discontinuities (detecting abrupt changes in intensity of image pixels) and similarity (detecting similarity in image pixels as per a set of predefined criterion) [13]. The taxonomy of the various techniques is elucidated in the subsequent sections.

\section{BOUNDARY BASED SEGMENTATION}

Partitioning an image into its constituent pixels by detecting abrupt changes in intensity of image pixels is marked as the boundary based segmentation approach. It is the most common approach for detecting meaningful discontinuities in the gray level image and works by detecting edges in the image which are considered as local changes in the image intensity [12]. The boundary based segmentation techniques are further categorized into Edge based segmentation and Watershed segmentation.

\subsection{EDGE BASED SEGMENTATION}

Spatial masks are used to detect edges in an image. Since these points, lines and edges are all high frequency components and therefore high pass masks are needed, for which the sum of the coefficients of the mask has to be equal to zero $[13,14]$. 
- Point Detection: It is used to detect the difference between a single pixel and the adjacent pixel. If at a location of mask, $|R| \geq T$, it implies detection of a point at that location, where $R$ is defined by (1) :

$\mathrm{R}=\mathrm{w}_{1} \mathrm{Z}_{1}+\mathrm{w}_{2} \mathrm{Z}_{2}+\ldots \ldots . .+\mathrm{w}_{9} \mathrm{Z}_{9}=\sum_{i=1}^{9}$ wizi

$\mathrm{T}$ is the threshold value2.

- Line Detection: The working of the line detection is similar to point detection and is used to detect the lines in an image. Since in an image, the lines may be in any direction, so to detect these lines, different masks like horizontal $\left(0^{\circ}\right), 45^{\circ}$, vertical $\left(90^{\circ}\right),-45^{\circ} / 135^{\circ}$ are used.

- Edge Detection: Edge is a set of connected pixels that lie on the boundary between two regions [9].

The algorithms for edge based operators include Laplacian, Zero-crossing, Prewitt, Sobel, Canny [4] etc. Edge detection operators are unable to find the exact edges. This segmentation method is very sensitive to noise i.e. usually suitable for images that are simple and noise free [14].

\subsection{WATERSHED SEGMENTATION}

Watershed segmentation algorithm is the discrete Vincent-Soille flooding watershed algorithm [17]. In this algorithm, an analogy is made between image and its constituent objects with watershed (ridge) and various catchment basins in it flooded with water. The output of this algorithm is the input image into its different catchment basins signifying objects in image and each basin is characterized by a unique label [16].

This technique produces more stable segmentation results and is faster than edge based techniques. But continuous boundaries between the regions can be obtained only when the watershed lines form connected paths [10]. Problem of over segmentation is there due to the noise. A large number of segmented regions are sometimes obtained unnecessarily [23].

\section{THRESHOLD BASED SEGMENTATION}

This technique considers the image as an arrangement of background and foreground and it takes the graylevel histogram of the image into account in which the pixels of the object and the backgrounds are divided into two principal forms . To extracts the objects from the background, a threshold ' $T$ ' is selected that separate these modes [18]. Any pixel $(x, y)$ of the image $f(x, y)$ for which the value of intensity is greater than or equal to ' $T$ ' is considered to be the foreground else it is considered to be the background[14]. Therefore the segmented image $\mathrm{g}(\mathrm{x}, \mathrm{y})$ is given by (2):

$$
\mathrm{g}(\mathrm{x}, \mathrm{y})=\left\{\begin{array}{l}
1 ; \text { if } \mathrm{f}(\mathrm{x}, \mathrm{y})>\mathrm{T} \quad \text {; for object } \\
0 ; \text { if } \mathrm{f}(\mathrm{x}, \mathrm{y})<\mathrm{T}
\end{array}\right.
$$

Based on the selection of threshold value, the thresholding may be of various types [11]:

- Global Thresholding: When value of $\mathrm{T}$ is a constant applicable over an entire image.

- Local/regional Thresholding: When value of $\mathrm{T}$ at any point $(\mathrm{x}, \mathrm{y})$ in an image depends on properties of a neighborhood of (x, y).

- Variable Thresholding: If value of $\mathrm{T}$ in not consistent, instead varies throughout the image.

- Dynamic/Adaptive Thresholding: When T depends on the spatial coordinates (x, y) themselves.

- Multiple Thresholding: this is useful for image with two different kinds of object of lighter intensity on a common background of darker intensity. Thus the segmented image is given by (3):

$$
\mathrm{g}(\mathrm{x}, \mathrm{y})=\left\{\begin{array}{lll}
\mathrm{p} & ; \text { if } \mathrm{f}(\mathrm{x}, \mathrm{y})>\mathrm{T}_{2} & \text {; for other object } \\
\mathrm{q} & \text { if } \mathrm{T}_{1}<\mathrm{f}(\mathrm{x}, \mathrm{y}) \leq \mathrm{T}_{2} & \text {; for one object } \\
\mathrm{r} & ; \text { if } \mathrm{f}(\mathrm{x}, \mathrm{y}) \leq \mathrm{T}_{1} & \text {; for background }
\end{array}\right.
$$

where $\mathrm{p}, \mathrm{q}, \mathrm{r}$ are three intensity values.

The threshold based technique finds its roots back to 1997, and the algorithm was proposed by Otsu [6] This technique overcame the shortcomings of boundary based segmentation and its variants are computationally less expensive; but don't engross spatial information of the images; and hence it causes noise, blurred edges or outlier in the image [1,3]. 
Further, all the thresholding algorithms consider only the point attributes of pixels and perform well only for images which have only two components/thresholds. Performance of thresholding algorithms decays for complex images, where more than two thresholds are present [1].

\section{REGION BASED SEGMENTATION}

To counter the drawbacks of thresholding, region based segmentation techniques evolved to be relatively simple and immune to noise $[1,14]$. These techniques work by dividing an image into regions which are alike as per a set of predefined criteria [13]. Region based segmentation is further divided into four modes viz.-a-viz. Region Growing, Region Merging, Region Splitting and Split and Merge.

\subsection{REGION GROWING}

Region growing works by gathering pixels with similar properties to form a region. It requires a seed pixel to start with. The connected pixels are then merged with the seed pixel around the seed pixel domain [19, 20]. Region growing can be processed into following steps:

- Select a seed pixel i.e. an arbitrary pixel (x,y) from the image that is to be segmented.

- Select a set of criteria for determining similar seeds based on properties like pixel intensity, gray scale texture, or color [26].

- Examine the nearest neighbours (4 or 8 neighbours) depending on 4-connectivity or 8-connectivity one by one.

- Grow the region by merging to seed which is similar to it on the basis of properties like pixel intensity, gray scale texture, or color.

The merging process terminates if there are no more pixels that match the similarity criteria. These algorithms are easy to complete and compute, but they are not used alone. Moreover, they need human intervention to select seed point. Patterns of regional growth are also sensitive to noise [20].

\subsection{REGION SPLITTING}

Region Splitting is also known as the Top-Down Approach. In this method, homogeneity property is satisfied where similar pixels are grouped together. The process starts by considering complete image as one region [19]. If the homogeneity property is satisfied, there is no more splitting, else the region is divided into four equal quadrants. This is repeated until all the regions satisfy the property. Generally each region is called a node. The parent node is split into its four (children) leaf nodes and so on, if it doesn't satisfy the given property.

\subsection{REGION MERGING}

Image segmentation may also be performed by an initial over segmentation results followed by the amalgamation of analogous neighboring regions into new regions on the basis of some predefined merging criteria [21]. The first step in region merging process (the bottom-up approach) is to either consider each pixel of the image as a region or partition the image into homogeneous regions using low-level image segmentation algorithm like watershed, etc. [22]. The next step involves representing each segmented region using attributes like intensity, color, texture, edge etc. After this representation, merging criterion is decided for all the pair of adjacent segmented regions and on that basis a pair or multiple pairs of adjacent regions are selected to merge into new region. The region merging process terminates until there is no pair of homogeneous region. To record the merging sequence, tree or graph representations may be utilized for e.g. binary partition tree, region adjacency graphs, etc. [1].

\subsection{SPLIT AND MERGE}

Region splitting and merging is a developing algorithm in image segmentation [24] proposed firstly by

Pavlidis [25]. The algorithm of Region splitting and merging consists of following steps:

- Splitting Step:

- A criterion is chosen for splitting the image based on quad tree [12].

- The image is split till each node (sub-node) has the same value of pixels.

- At the same time, the number of splitting levels can be determined. 
- Merging Step: If the adjacent regions satisfy the similarity or homogeneity properties, they are merged together.

- Repeat merging until no further merging is possible.

The region split and merge technique is a combination of top-down and bottom-up approach and is very simple in nature. It also gives dynamic solution to image segmentation and is based on a specific region model described by a homogeneity predicate [15]. Better segmentation quality is provided by this approach owing to increase in computational speed with less complexity $[12,31]$ and connected regions are also ensured. But this technique is less adaptive to image characteristics i.e. it produces large number of region boundaries, other than the horizontal and vertical ones [19]. This shortcoming has been countered by the applying optimization techniques either locally or globally [26].

\section{OPTIMIZING IMAGE SEGMENTATION}

Optimization techniques are used as a set of auxiliary tools that may be used at any step of image processing chain. Eventually many algorithms have been proposed in the past that intersperse segmentation with optimization to yield better results [27]. The following section articulates the optimization algorithms for segmenting an image into its constituent objects.

\subsection{SNAKES/ACTIVE CONTOURS}

The active contour method provides an effective way for segmentation, which identifies object boundaries by evolving computer generated curves that move within image under the influence of internal and external forces [28]. Active contour/Snake is placed near the boundary of region of interest (ROI). Various forces influence the snake towards the target during the iterative process. The location and contour of the snake within the image is controlled by the forces. An energy function is that measures the appropriateness of contours of ROI and minimization of this energy function represents active contour's total energy [28]. Although a traditional method to minimize energy and optimize the results but this method requires user interaction for an initial placement of snake. It is also sensitive to noise and choice of its parameters offering a high computational complexity [23].

\subsection{PARTICLE SWARM OPTIMIZATION}

Optimization is applied to reduce the complexity of segmentation and Particle Swarm Optimization (PSO) has also sustained as an efficient technique to obtain optimized value of threshold for segmentation of an image. It was proposed by Kennedy and Eberhart in 1995 and is a population-based stochastic optimization algorithm [34]. As compared to other optimization tools, PSO has a better search performance with faster and more stable convergence rates [36]. PSO imitates the food searching conduct of birds flock. Each pixel of the image or the so-called search space represents a candidate solution. Each pixel is assigned at random, a position and velocity and candidate solutions are updated by changing the velocity and position of the particles [34]. This algorithm can be differentiated into global-best PSO (g-best PSO) and local-best PSO (1-best PSO). Update of candidate best position of a particle is subject to the entire swarm in the case of global-best PSO. And in case of local-best PSO first entire swarm is divided into some overlapping particles and on the basis of position of other particle/pixel in the region, best particle is chosen [35]. Each particle's movement is affected by its known local best position and also guided by the global best position [32].

\subsection{GENETIC ALGORITHM BASED SEGMENTATION}

A powerful statistical search method, Genetic algorithm (GA) can be used in an integrated manner with traditional image segmentation techniques for detection of edges in an image. It considers the grouping of each pixel into other pixels by distance functions based upon segments computed already by traditional segmentation technique. The initial population thus generated is evaluated by a fitness function which is defined on the basis of distance function computed between segments also denoted as chromosomes in GA terminology. In this evaluation process, if the maximum segmentation quality for current population is above a predefined threshold, the GA cycle 
terminates else the best solutions are subject to either crossover or mutation, depending upon the application. GA based segmentation provides more accurate results than traditional methods, faster convergence but overall processing time is high which is disadvantageous. Moreover, the visual quality of segmentation is not up to the mark [29].

\subsection{CLUSTERING BASED SEGMENTATION}

Classification of pixels into one or the other cluster/categories in order to maximizing the intra-class similarity and minimizing the inter-class similarity of pixels may be implemented to segment an image [30]. To define the similarity feature any attribute of image may be chosen for the same like intensity of pixels, distance of pixel intensities, etc. then similar pixels are grouped together to form clusters. Clustering algorithms are classified as k-means, fuzzy clustering, hard clustering, etc. These algorithms are simple and computationally less expensive but require a priori knowledge of number of clusters and are sensitive to noise. These are also susceptible to variations in intensity. These drawbacks are overcome by the fuzzy c-means clustering algorithm [2].

\subsection{ARTIFICIAL NEURAL NETWORKS}

Besides their phenomenal applications in various real life applications like data processing, robotics, fitness approximation, computer numerical control, etc., neural networks have also advanced in image processing. Mapping image into a Neural Network where each pixel corresponds to a neuron, training of the network is done to determine the connection and weights between nodes. Segmentation of new images is done with the trained neural network [27]. A prerequisite to this method is feature extraction which is followed by energy minimization. This technique has high parallel ability, fast computing ability and high immunity to noise. But initialization of network affects the result of segmentation and training of the network is an exaggerated process [23].

\section{QUANTITATIVE ANALYSIS}

The techniques discussed above affect differently depending upon the type of images they act upon. The evaluation of segmentation may be either subjective or objective [37]. The subjective evaluation is based on the analysis done by group of humans and is generally termed as the qualitative analysis whereas the objective evaluation is a quantitative analysis on the basis of some metrics. The objective evaluation may be further exploited to measure intra-algorithmic features or to compare two different segmentation techniques. Analytical survey for one of each category of segmentation on the latter in the MATLAB workspace is extended presently on a gray scale image of human skull (fig.4.) of pixel size 512*512.

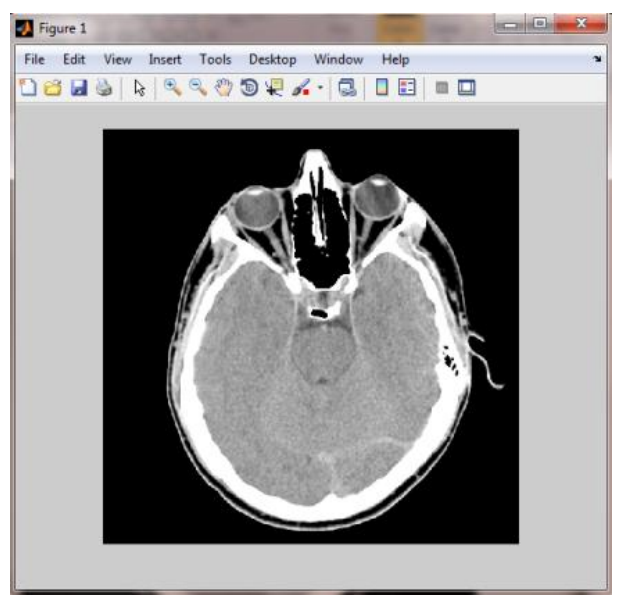

Fig.4: Gray scale Image of length $*$ width $=512 * 512$ pixels. 
Mean Square Error (MSE) and Peak Signal-to-Noise Ratio (PSNR) are used as the metrics to assess the quality of segmented image from various segmentation techniques with respect to the original image. MSE represents the cumulative squared error between the segmented and the original image, whereas PSNR represents a measure of the peak error. The lower the value of MSE, the lower the error and higher the PSNR, the better is the quality of segmented image [38, 39].

Fig. 5 shows the segmentation of image of fig. 4 by the thresholding techniques using the Otsu method [6] and segmentation using splitting of image is shown in fig.6. Fig. 7 depicts the segmented output of split and merge segmentation technique, The value of MSE is highest for 'split and merge' region based segmentation and eventually the PSNR is least which is undesirable. Segmenting the image with particle swarm optimization results in lowest value of MSE and is shown in fig.8. Table 1 shows the metric evaluation of various algorithms and it is clearly evident that optimization produces better quality of segmentation result which is evident through the visual assessment of the results.

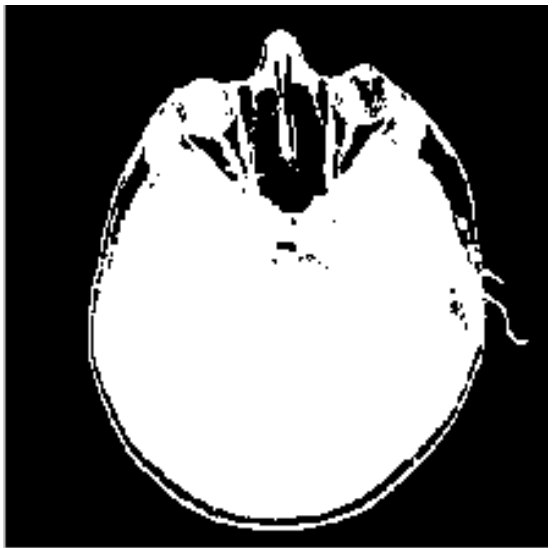

Fig. 5: Thresholding using Otsu method.

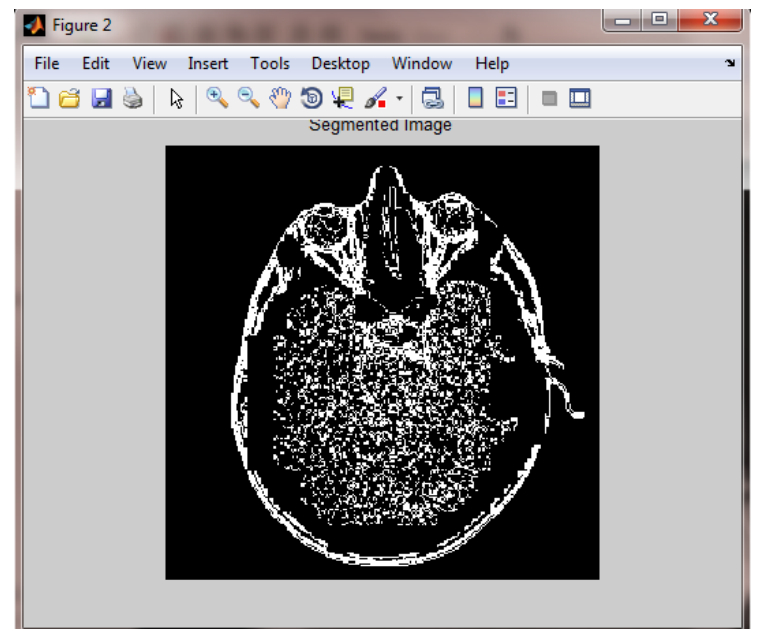

Fig.7: Split and merge using region based segmentation

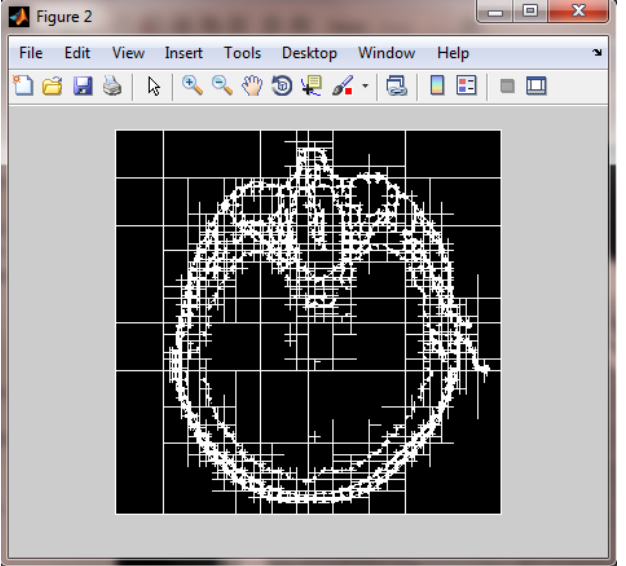

Fig.6: Splitting using region based segmentation.

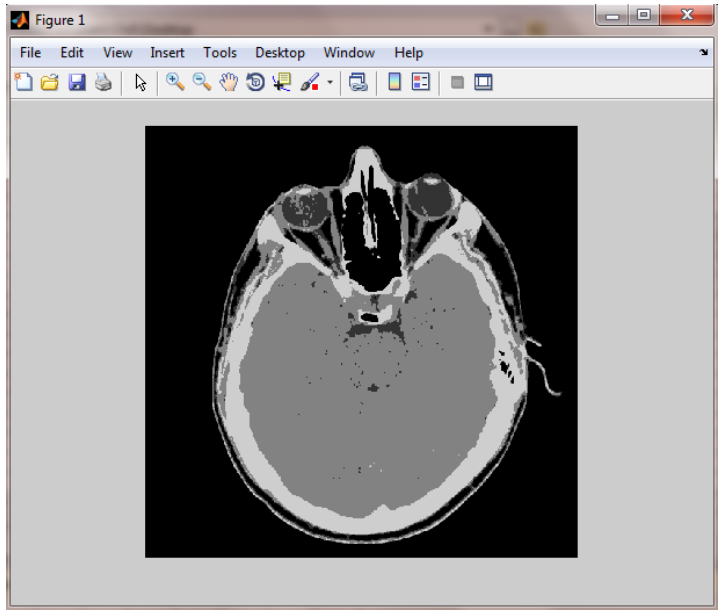

Fig. 8: Particle swarm optimization

TABLE I. Metric analysis of various segmentation techniques

\begin{tabular}{|l|c|c|}
\hline Segmentation Technique & $\begin{array}{c}\text { MSE } \\
\text { (dB) }\end{array}$ & $\begin{array}{c}\text { PSNR } \\
\text { (dB) }\end{array}$ \\
\hline Thresholding using Otsu method & 83.42 & 12.48 \\
\hline
\end{tabular}




\begin{tabular}{|l|l|l|}
\hline Splitting & 57.14 & 25.61 \\
\hline Split and Merge & 85.44 & 11.47 \\
\hline Particle Swarm Optimization & 56.22 & 26.66 \\
\hline
\end{tabular}

\section{CONCLUSION}

Development of diverse innovative anthology of segmentation methods used for various real-life applications have been cited in this article. Each technique offers a set of advantageous features but with some tradeoffs. This has led to confinement of each method being suitable for particular types of images. Although, the quantitative assessment of the various segmentation techniques reveals the better performance of applying optimization but in the due course, there has been no such method that could be adopted universally for the segmentation of all types of images. This will certainly pave way for further research in this domain for the evolution of a unanimous technique.

\section{REFERENCE}

[1] W.X. Kang, Q. Yang and R.P. Liang, The comparative research on image segmentation algorithms, IEEE First International Workshop on Education Technology and Computer Science, 2009, 703-707.

[2] M.A. Ali, L.S. Dooley and G.C. Karmakar, Object based image segmentation using fuzzy clustering, Proc. IEEE Conf. on Acoustics, Speech and Signal Processing, France, 2006,105-108.

[3] R. Yogamangalam and B. Karthikeyan, Segmentation techniques comparison in image processing, International Journal of Engineering and Technology, 5(1), 2013, 307-311.

[4] J. Canny, A computational approach to edge detection, IEEE Trans. Pattern Analysis and Machine Intelligence, 8(6), 1986, 679 - 698.

[5] V.V.D. Shah, Image processing and its military applications, Defense Science Journal, 37(4), 1987, 457-468.

[6] N. Otsu, A threshold selection method from gray level histograms, IEEE Trans. Systems, Man and Cybernetics, 9(1), 1979, 62-66.

[7] E.S. Gopi, Digital signal processing for medical imaging using MATLAB (New York: Springer, 2013).

[8] H. Pandit and D.M. Shah, Application of Digital image processing and analysis in healthcare based on medical palmistry, Proc. Int. Conf. on Intelligent Systems and Data Processing, 2011, 56-59.

[9] R. Harle and M.R. Joshi, Review on image segmentation methods, International Journal of Computer Science and Mobile Computing, 3(4), 2014, 722-726.

[10] D.P. Patil and S. G. Doere, Medical image segmentation: A Review, International Journal of Computer Science and Mobile Computing, 2(1), 2013, 22-27.

[11] A. Fakhri, A. Nasir, M. Nordin, A. Rahman and A.R. Mamat, A study of image processing in agriculture application under high performance computing environment, International Journal of Computer Science and Telecommunications, 3(8), 2012,16-24.

[12] P. Thakare, A study of image segmentation and edge detection techniques, International Journal of Computer Science and Engineering, 3(2), 2011, 899-904.

[13] R.C. Gonzales and R.E. Woods, Digital image processing (India: Pearson Education, 2009).

[14] R. Kandwal, A. Kumar and S. Bhargava, Review: existing image segmentation techniques, International Journal of Advanced Research in Computer Science and Software Engineering, 4(4), 2014, 153-156.

[15] H.G. Kaganami and Z. Beiji, Region-based segmentation versus edge detection, Proc. $5^{\text {th }}$ Int. Conf. on Intelligent Information Hiding and Multimedia Signal Processing, 2009, 1217-1221.

[16] C.M. Pun, N.Y. An and M. Cheng, A region-based image segmentation by watershed partition and dct energy compaction, Proc. IEEE Conf. on Computer Graphics, Imaging and Visualization, China, 2011, 131-135.

[17] L. Vincent and P. Soille, Watersheds in digital spaces: an efficient algorithm based on immersion simulations, IEEE Trans. Pattern Analysis and Machine Intelligence, 13(6), 1991, 583-598.

[18] P.D.R. Raju and G. Neelima, Image segmentation by using Histogram Thresholding, International Journal of Computer Science and Telecommunications, 2(1), 2012, 776-779.

[19] K. Khoshelham, Z. Li and B. King, A Split-and-Merge Technique for Automated Reconstruction of Roof Planes, Photogrammetric Engineering \& Remote Sensing Journal, 71(7), 2005, 855-862.

[20] J. Tang, A color image segmentation algorithm based on region growing, Proc. $2^{\text {nd }}$ IEEE. Conf. on Computer Engineering and Technology, 2010, 6, 634-637.

[21] B. Peng, L. Zhang and D. Zhang, Automatic image segmentation by dynamic region merging, IEEE Trans. Image Processing, 20(12), 2011, 3592-3605.

[22] L. Zha, Z. Liu, S. Luo and L. Shen, A novel region merging based image segmentation approach for automatic object extraction, IEEE International Symposium, Beijing, 2013, 970-973.

[23] R. Dass, Priyanka and S. Devi, Image segmentation techniques, International Journal of Electronics \& Communication technology, 3(1), 2012, 66-70. 
[24] R. Xiang and R. Wang, Range image segmentation based on split-merge clustering, Proc. IEEE Conf. on Pattern Recognition, Beijing, 2004, 614-617.

[25] S.L. Horowitz and T. Pavlidis, Picture segmentation by a tree traversal algorithm, Journal of the Association for Computing Machinery, 23(2), 1976, 368-388.

[26] A.B.M. Faruquzzaman, N.R. Paiker, J. Arafat, Z. Karim and M.A. Ali, Object segmentation based on split and merge algorithm, Proc. IEEE Conf. on Acoustics, Speech and Signal Processing, France, 2006, 1-4.

[27] M.E. Petersen, D. Ridder and H. Handels, Image processing with neural networks, The Journal of the Pattern Recognition Society, Elsevier, 35, 2002, 2279-2301.

[28] T.N.A. Nguyen, J. Cai, J. Zhang and J. Zheng, Robust interactive image segmentation using convex active contours, IEEE Transactions on Image Processing, 21(8), 2012, 3734-3743.

[29] A. Sheta, M.S. Braik and S. Aljahdali, Genetic algorithms: a tool for image segmentation, Proc. Int. Conf. on Multimedia Computing and Systems, 2012, $84-90$.

[30] M. Xinyan, Z. Ying, H. Yanxiao and S. Binjie, Color image segmentation method based on region growing and ant colony clustering, Global Congress on Intelligent System, 2009, 173-177.

[31] D. Kelkar and S. Gupta, Improved Quadtree Method for Split Merge Image Segmentation, Proc. of IEEE Conf. on Emerging Trends in Engineering and Technology, Maharashtra, 2008, 44-47.

[32] F. Jiang, M. R. Frater and M. Pickering, Threshold-based image segmentation through an improved particle swarm optimization, Proc. IEEE Conf. on Digital Image Computing Techniques and Applications, 2012.

[33] S. Banerjee and N. D. Jana, Bi Level Kapurs Entropy based Image Segmentation using Particle Swarm Optimization, Proc. IEEE Conf. on Computer, Communication, Control and Information Technology, 2015.

[34] J. Kennedy and R. Eberhart, A discrete binary version of the particle swarm algorithm, Proc. IEEE Conference on Systems, Man, and Cybernetics, Computational Cybernetics and Simulation, 5, 1997, 4104-4108.

[35] J. Kennedy and R.C. Eberhat, Swarm Intelligence, San Francisco, USA, Academic Press, 2001.

[36] J. Kennedy and R. Eberhart, Particle swarm optimization, Proc. IEEE Conf. on Neural Networks, 4, 1995, 1942-1948.

[37] Y.J. Zhang, A survey on evaluation methods for image segmentation, Elsevier Journal on Pattern Recognition, 29(8), 1999, 1335 -1346.

[38] M. Sharma and V. Chouhan, objective evaluation parameters of image segmentation algorithms, International journal of engineering and advanced technology, 2, 2012, 84-87.

[39] H. Zhang, J.E. Fritts, S.A. Goldman, Image segmentation evaluation: A survey of unsupervised methods, Science Direct Computer Vision and Image Understanding, 110, 2008, 260-280. 